\title{
Research on deer house monitoring system based on Internet of things Hongyan Ju' ${ }^{a}$, Shijun Li and He Gong School of Jilin Agricultural University, Changchun 130118, China. \\ adidiaoxiaoju@163.com
}

Keywords: Environmental parameters, sensors, ZigBee, early warning.

\begin{abstract}
There is a high requirement for the growth environment in the deer breeding, Have a great impact on the antler growth, especially temperature, humidity, illumination and other environmental parameters. This study designs a deer house monitoring system based on Internet of things, able to monitor and display parameters including temperature, humidity, and light in time of the deer's house, to monitor the physical condition of a deer, including deer's body temperature and body weight, complete monitoring and early warning function. To reduce labor costs and improve production efficiency.
\end{abstract}

\section{Introduction}

The sika deer belong to artiodactyla in medium-sized deer, its $140-170 \mathrm{~cm}$ length in, $85-100 \mathrm{~cm}$ in height, weight between 100-150 kg. Only the stag has horns, usually four forks. Because the body with white spots, looks like the plum blossom, so they are called sika deer. Hereinafter referred to as deer. The deer body is a treasure, including Antler, penis cervi, deer placenta, deer blood and so on, all of these have high medicinal value and practical value. Antler is the most precious thing on the body of a deer, the nutritional composition of pilose antler is very rich, is the best medicine as one of the three treasures in the northeast region of China. Deer farming is mainly distributed in the northeast three provinces in China, people in Jilin province to the development of breeding deer as a characteristic industry. The local government has introduced many policies to support the development of deer. In 2011 the number of deer in Jilin has reached 50\% of the country's total [1]. The deer have very high demand in the process of deer in the growth environment, especially temperature, humidity, light and other environmental influences on the antler growth greatly, it is very important to know the deer growth environment and do the right response in time. As early as in the 80 s of last century, scientists in Jilin province conducted a detailed study on the effects of environmental parameters on the growth of deer [2-3]. In recent years, there are a lot of research on how to improve the yield of antler [4-6].

\section{Experimental materials}

\subsection{Experimental base}

The experimental deer farm is Jilin Province DONGDAO deer industry science and Technology Development Co. Ltd. which is in Jilin province Changchun Shuangyang Economic Development Zone, covers an area of 72 thousand square meters, it is a comprehensive "Production, Learning, Research," " Farming, Machining, Sale " as a whole deer industry development group.

\subsection{Experimental equipment}

Experimental equipment, including temperature and humidity sensor DHT91, light sensor SM2161B, ZigBee wireless module JN5168, infrared thermometer Raytek CM, pressure sensor 154N, LED display, LED driver module TLC5941 and so on. 


\section{Experimental methods}

The overall design of the monitoring system based on the Internet of things is shown in figure 1. The whole system is divided into four parts, including the deer's physical condition information collection, the collection of environmental parameters, the data transmission and the display warning.

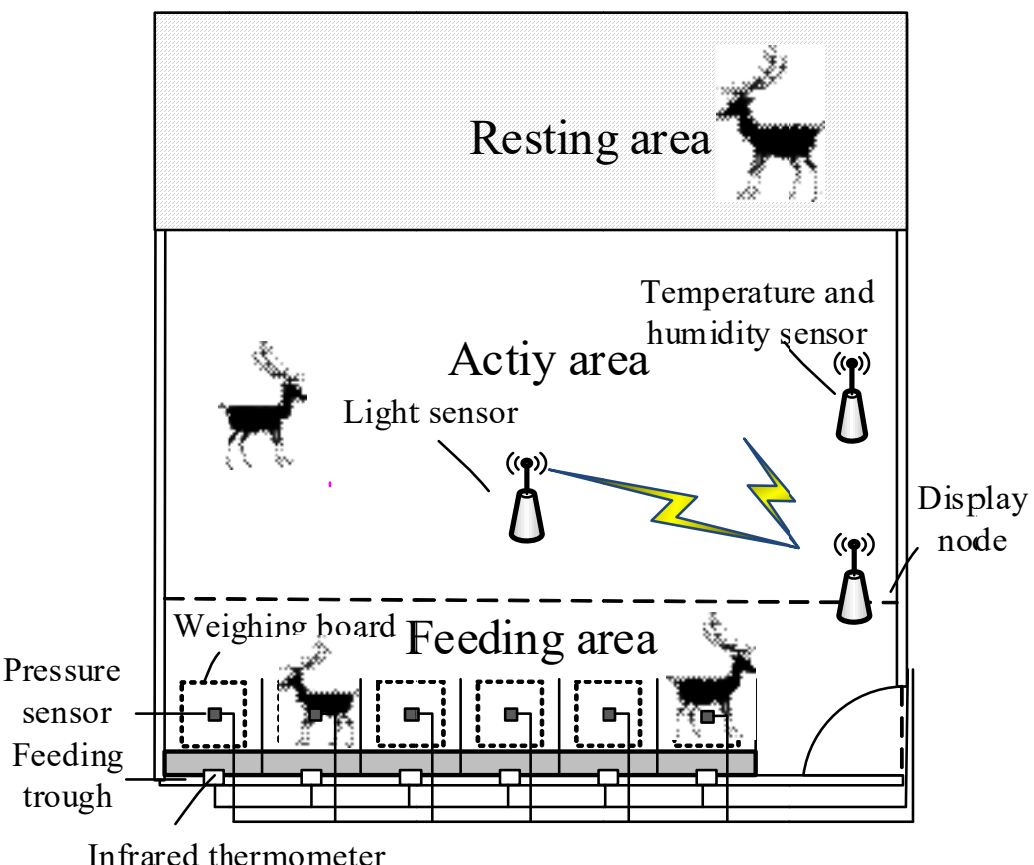

Fig. 1 Deer house monitoring system based on Internet of things.

\subsection{Deer's physical condition information collection}

In this part, the data of deer growth status were collected, and the system was equipped with deer weight data acquisition and temperature data acquisition. In this system, the infrared thermometer Raytek CM is used to collect the deer's body temperature.

Table 1 The characteristics of Raytek CM infrared thermometer.

\begin{tabular}{|c|c|c|c|c|}
\hline $\begin{array}{c}\text { measuring } \\
\text { range }\left({ }^{\circ} \mathrm{C}\right)\end{array}$ & $\begin{array}{c}\text { Optical } \\
\text { resolution(dpi) }\end{array}$ & $\begin{array}{c}\text { response } \\
\text { time }(\mathrm{ms})\end{array}$ & $\begin{array}{c}\text { Stainless steel } \\
\text { shell }\end{array}$ & Seal grade \\
\hline$-20 \sim 500^{\circ} \mathrm{C}$ & $13: 1$ & 150 & NEMA-4X & IP65 \\
\hline
\end{tabular}

Infrared thermometer Raytek CM has many advantages, including a wide range of measurement, high precision, small size, with stainless steel shell, etc., it is a new generation of infrared thermometer, which is the most powerful but small size products in the market. It uses the RS232 digital communications interface, dedicated WINDOWS operating software for the operator to set the remote parameters, and it can simultaneously monitor multiple probes for the remote monitoring of the probe provides great convenience.

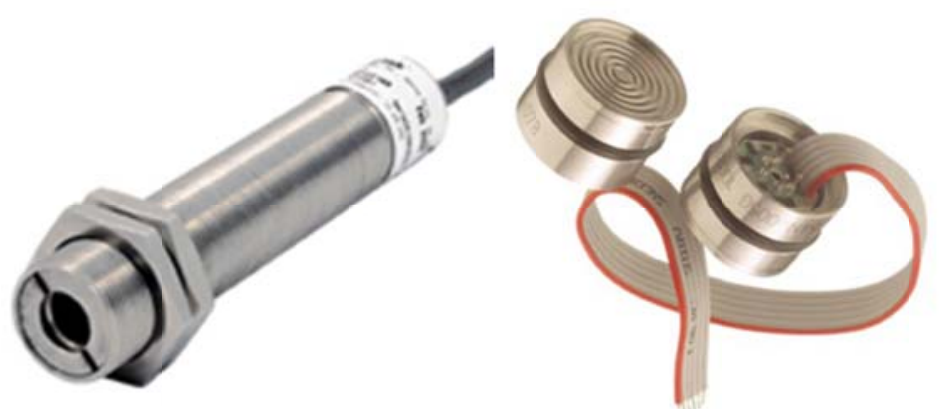

Fig. 2 Raytek CM infrared temperature instrument and 154N-001G-RT pressure transducer.

The pressure sensor is a 154N-001G-RT pressure sensor, which has the advantages of low power consumption, solid state structure, reliable performance, sealing and so on. 
Table 2 The characteristics of Raytek CM infrared thermometer.

\begin{tabular}{|c|c|c|c|c|c|}
\hline $\begin{array}{c}\text { Measuring } \\
\text { range (Psi) }\end{array}$ & $\begin{array}{c}\text { Compensation } \\
\text { temperature } \\
\left({ }^{\circ} \mathrm{C}\right)\end{array}$ & $\begin{array}{c}\text { Full scale } \\
\text { output }\end{array}$ & $\begin{array}{c}\text { Zero } \\
\text { output }\end{array}$ & Nonlinear & $\begin{array}{c}\text { Stainless } \\
\text { steel shell }\end{array}$ \\
\hline $0 \sim 5$ & $-40 \sim 125$ & $50 \sim 100$ & $-2 \sim 2$ & $-0.2 \sim 0.2$ & 316 \\
\hline
\end{tabular}

The 154N-001G-RT pressure sensor is a kind of silicon piezoresistive sensor which is compensated and compatible with the medium. The shell is made of 316 stainless steel. The external pressure is transferred to the sensing element of the sensor through the 316 stainless steel membrane and the internal filling silicone oil.

The design of the deer house structure as shown in Figure 1, is separated by a fence in the feeding trough, and only one deer is allowed to eat in each compartment to ensure that only one deer is tested at each interval. Each compartment is set to $110 \mathrm{~cm}$ in this experiment. An infrared thermometer is arranged on the wall above the feeding trough of each section, and is about $40 \mathrm{~cm}$ from the feeding trough. A weighing board is arranged below the feeding trough, and is hard board, wide $100 \mathrm{~cm}$, long $180 \mathrm{~cm}$, a wide side of the weighing board edge in the feeding trough below, two long side from the fence in $5 \mathrm{~cm}$, the weighing board is connected with the pressure sensor. The system can measure the deer's body temperature and body weight when the deer eats the food, and the data measured by the infrared thermometer and the pressure sensor will be transmitted to the display screen through the wire.

Because the installation position of the pressure sensor and infrared thermometer is relatively low in the deer house, taking into account the possible problems, such as rain, spray, urine or other factors will affect the normal operation of the equipment, So choose the sensor to consider its tightness, corrosion resistance, reliability, and ultimately choose Raytek CM infrared thermometer and $154 \mathrm{~N}-001 \mathrm{G}-\mathrm{RT}$ pressure sensor to monitor the body temperature and weight of the deer.

\subsection{Collection of environmental parameters}

The environmental parameters are collected by the sensors distributed in the deer house, including temperature, humidity and illumination. The collected environment parameters are transmitted to the display screen by ZigBee wireless. At present, the commonly used digital temperature and humidity sensor has DHT series, HTU series, SHT series, taking into account the measurement range, precision and other factors to choose DHT91, its better performance, higher accuracy, moderate price.

Table 3 Characteristics of DHT91 temperature and humidity sensor

\begin{tabular}{|c|c|c|c|c|}
\hline $\begin{array}{c}\text { Temperature } \\
\text { measurement } \\
\text { range }\left({ }^{\circ} \mathrm{C}\right)\end{array}$ & $\begin{array}{c}\text { Humidity } \\
\text { measurement } \\
\text { range (RH) }\end{array}$ & $\begin{array}{c}\text { Temperature } \\
\text { accuracy }\left({ }^{\circ} \mathrm{C}\right)\end{array}$ & $\begin{array}{c}\text { Humidity } \\
\text { accuracy }(\mathrm{RH})\end{array}$ & $\begin{array}{c}\text { Response } \\
\text { time }(\mathrm{s})\end{array}$ \\
\hline $40-123.8$ & $0 \%-100 \%$ & \pm 0.4 & $\pm 3 \%$ & 4 \\
\hline
\end{tabular}

This system is designed with SM2161B light sensor monitoring, it is with the RS485 interface, the standard MODBUS-RTU protocol, can realize multi-point monitoring and network transmission, its large range and the maximum allowable error is far lower than other types of light sensors.

Table 4 Characteristics of SM2161B light sensor

\begin{tabular}{|c|c|c|c|}
\hline $\begin{array}{c}\text { Measuring range } \\
\text { (lux) }\end{array}$ & Accuracy & baud rate $(\mathrm{Bd} / \mathrm{s})$ & $\begin{array}{c}\text { Operating } \\
\text { temperature }\left({ }^{\circ} \mathrm{C}\right)\end{array}$ \\
\hline $0 \sim 200000$ & $\pm 7 \%$ & 9600 & $-40 \sim 85$ \\
\hline
\end{tabular}

The data collected by the sensor is transmitted to the display screen through the ZigBee wireless module JN5168. The following is the working principle diagram. 


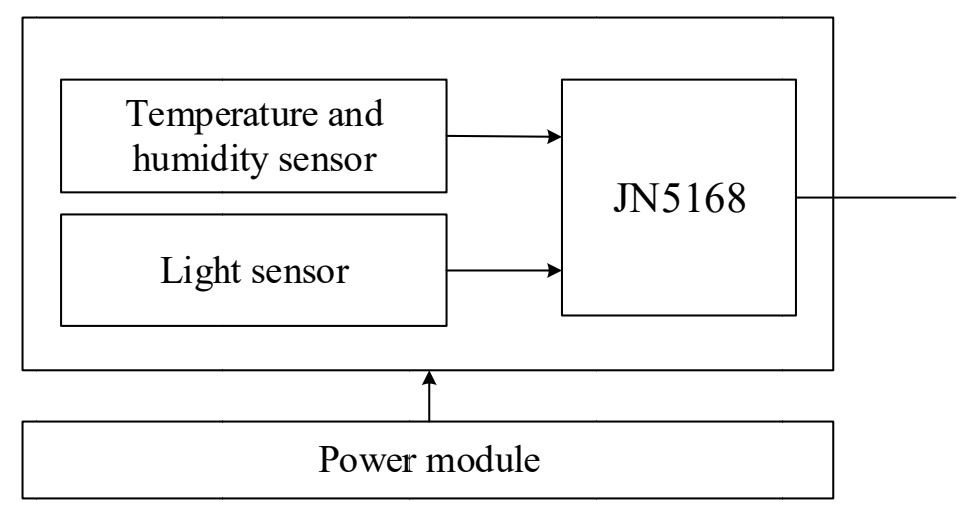

Fig. 3 The working principle of environmental acquisition

\subsection{Data transmission}

Data transmission using ZigBee wireless transmission, which is a short distance, selforganization, low power consumption, low complexity, low cost, can be embedded in variety of devices, etc.. JN5168 is a low power consumption and high performance module which is produced by Jennic. It has the advantages of low power consumption, low cost, rich peripheral interface and easy to develop.

The data transmission circuit diagram is shown in figure 5. Button SW1 for programming keys, resistor R1, capacitor $\mathrm{C} 1$ and key SW1 constitute reset circuit, DHT91 uses I2C interface communication, the JN5168 CLK pin and DATA pin are respectively connected to the SCK pin and the DATA pin of the DHT91. SM2161B light sensor using the RS485 communication interface, it can not be directly connected with the JN5168, it is necessary to pass through the level conversion module as an intermediate link.

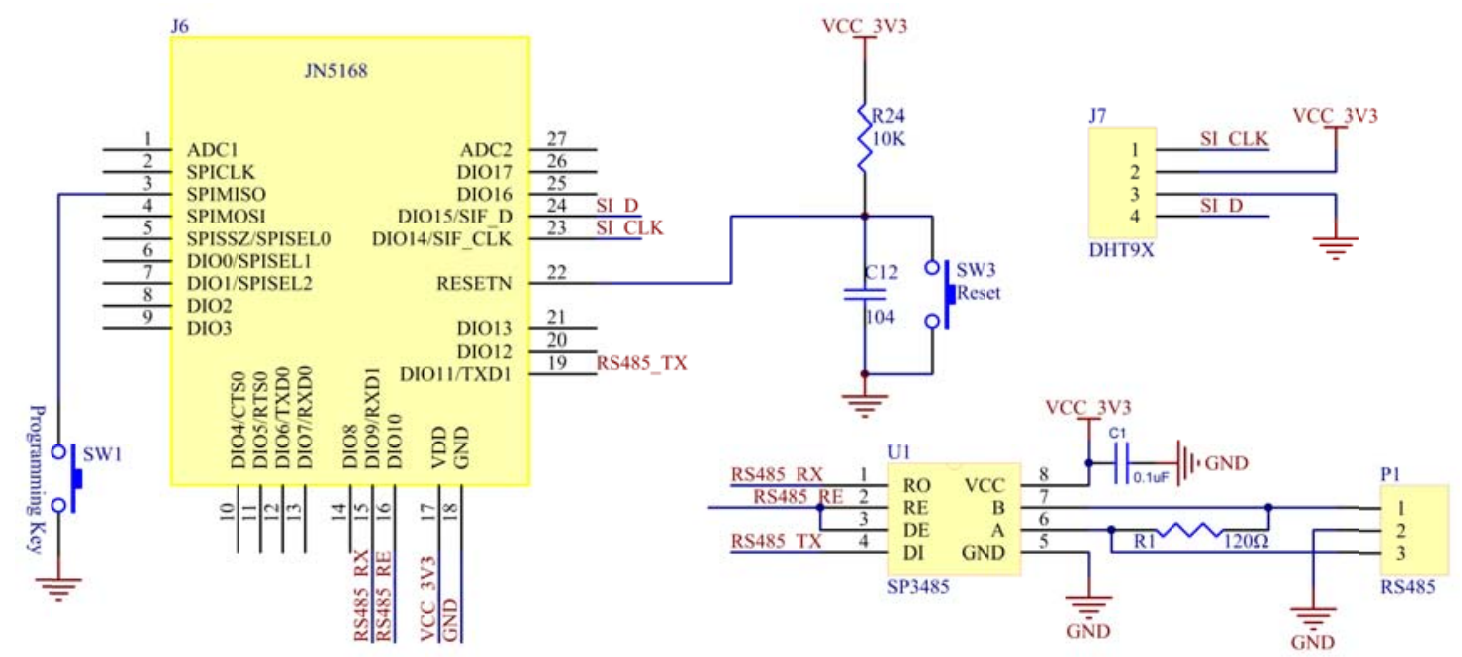

\subsection{Display warning}

Fig. 4 Data transmission circuit diagram

Display warning node is installed on the side wall of the deer house near the door, It is used to display temperature, humidity, light value from the deer house, at the same time, the data of body temperature and body weight of the deer are displayed, and when the deer body temperature exceeds the warning value, the utility model is displayed and the alarm is sent out by the buzzer.

LED driver chip is TLC5941 it has a total of 28 pins, it is a 16 channel constant current driver, it can drive 16 LED at the same time, all of its internal data registers, brightness registers, point correction registers and error status information are accessed through the serial interface, the maximum serial clock rate of 30MHz. Figure 6 shows part of the working principle diagram. 